\title{
Desde la clasificación de Kudo a la inteligencia artificial en endoscopía. Una historia de 30 años
}

\author{
Raimundo Izquierdo C. ${ }^{1}$, Luis Pastén $C .{ }^{l}$, Rodney Stock L. ${ }^{l}$, Alex Navarro R. ${ }^{l}$ y Roque Sáenz F. ${ }^{l}$
}

From Kudo classification to artificial intelligence in endoscopy. A 30-year history

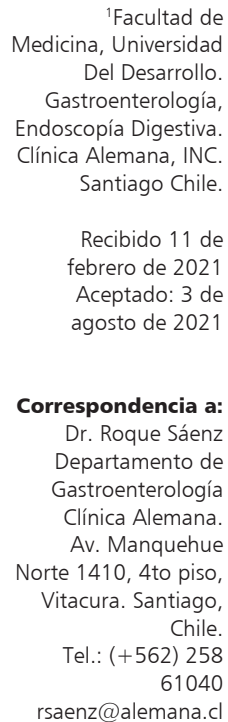

rsaenz@alemana.cl

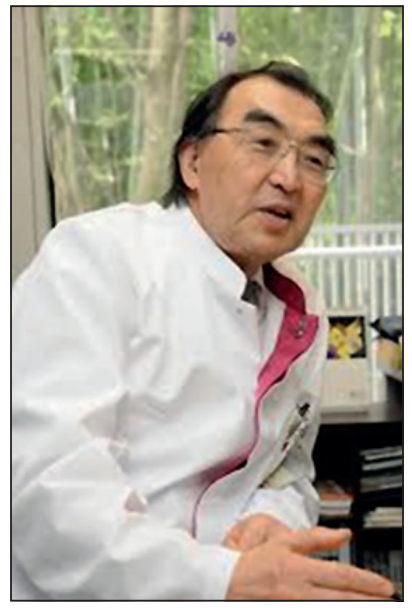

Shin-ei Kudo MD, PhD

Chairman of Digestive Disease Center- Showa University Northern Yokohama Hospital. Chairman of Showa University International Training Center for Endoscopy and Endoscopic Surgery (SUITE).

\section{Significados de "Kudo"}

- “La admiración pública que recibe una persona como resultado de un logro en particular o su posición en la Sociedad".

- "Elogio, alabanza, admiración y fama recibidos por un logro".

Estos conceptos aplican perfectamente al Profesor Kudo, quien es merecedor de un reconocimiento como pionero y líder visionario en Endoscopía Gastrointestinal, en el mundo entero.

Hace 30 años, se hizo realidad el sueño de hacer posible que una descripción endoscópica del Patrón de Criptas (PP-Pit Pattern), gracias a la magnificación y los avances electrónicos y computacionales como la cromo endoscopía (tinciones directas o electrónicas), más una concepción tridimensional de la mucosa, nos han llevado a obtener una visión "patológica" de las lesiones permitiendo diferentes acciones en consecuencia.

a. Lesiones no riesgosas.

b. Lesiones pre-malignas o malignas precoces que deben ser resecadas mayormente vía endoscópica $\mathrm{y}$,

c. Lesiones malignas y avanzadas, para enfrentamiento quirúrgico.

Estos conceptos que aparecen sustentados en las investigaciones y clasificación del Prof. Kudo, fueron adoptados rápidamente en la comunidad médica internacional y el autor fue reconocido en el mundo entero.

El sueño de obtener un diseño de las características de la microscopía y patología desde la endoscopia hecho una realidad.

Ha enseñado con entusiasmo tanto a endoscopistas de su país como extranjeros, viajando incansablemente como un "embajador de la endoscopía".

Es un profesor genuino y mentor de generaciones, conjugando alrededor de él, a un grupo de elite en la Universidad de Showa en Yokohama. (Showa University in Northern Yokohama Hospital), en el cual es Chair del Digestive Disease Center.

Ha ejercido numerosos cargos académicos y dirigenciales en instituciones de la especialidad tanto en Japón como en el mundo.

También es el jefe del "Showa University International Training Center for Endoscopy and Endoscopic Surgery". (SUITE). Un centro muy reconocido y Centro de Excelencia de la WEO. (World Endoscopy Organization) y seguramente uno de los mejores del mundo.

Él y su grupo son responsables de una gran cantidad de nuevo conocimiento, innovaciones y aplicaciones que están lejos de decaer, sino por el contrario, aparecen y nos ayudan constantemente y nos mantienen expectantes de recibir nuevos aportes. 
Para nombrar algunos de los logros:

- Más de 300.000 casos y 10 libros. Innumerables artículos de liderazgo en endoscopia en revistas de primer nivel.

\section{- The Kudo Classification (30 años).}

- Diagnostico endoscópico de CCR precoz y su tratamiento.

- CCR Deprimido 0-IIc, "De Novo Cancer".

- POEM, (Prof. Inoue).

- Lateral Spreading Tumors (LST) El concepto. (Granular y No granular).

- $E M R / E S D$.

- Endocystoscopy.

- Más recientemente, un gran impulso en el desarrollo de la endoscopia asistida por IA (Inteligencia Artificial) en gastroenterología y sus aplicaciones. Tecnología en expansión y que veremos reflejada en nuestro trabajo diario, en los años venideros. (Endo-Brain) (Figura 1).

Otros expertos en su grupo, desarrollando innovaciones y aplicaciones, además del Prof. Haruhiro Inoue, son, Mori Y, Hirasawa T, Misawa M, Takeda $\mathrm{K}$, Ichimasa $\mathrm{K}$, entre otros.

Estas son contribuciones sobresalientes a la ciencia y una perspectiva práctica para la endoscopia digestiva. La endoscopia permite un enfoque patológico preciso según sus aportes en nuestro trabajo cotidiano (Figura 2).

Expresamos nuestra gratitud y reconocimiento por sus aportes a la ciencia y a la Endoscopia Digestiva en particular, como un legado de toda su vida (Figuras 3 y 4$)$.

El Profesor Shin-ei-Kudo, Merece nuestro reconocimiento y admiración.

\section{KUDOS FOR KUDO SENSEI!!}
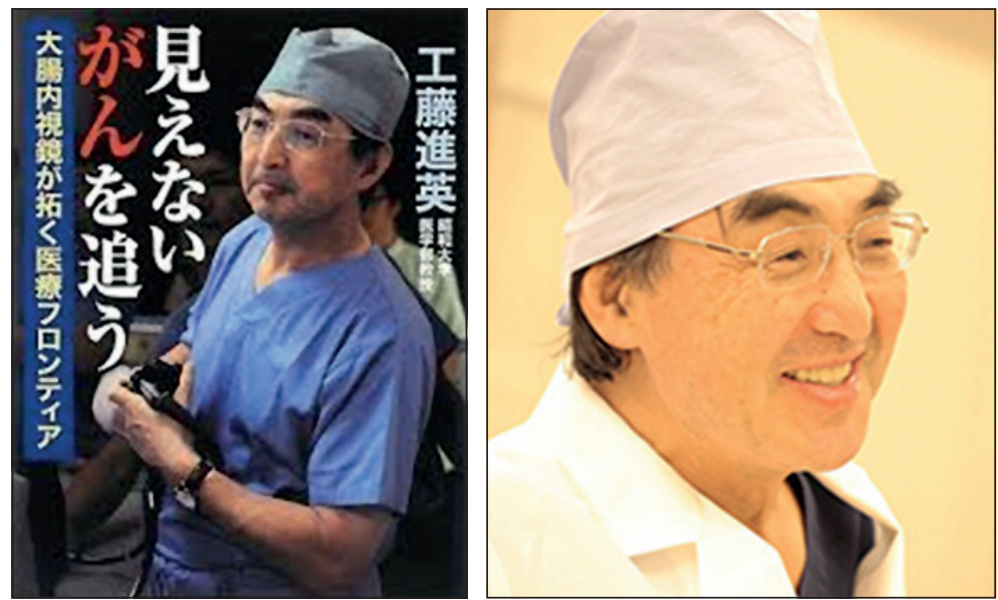

Figura 1. Profesor Shin-ei Kudo.

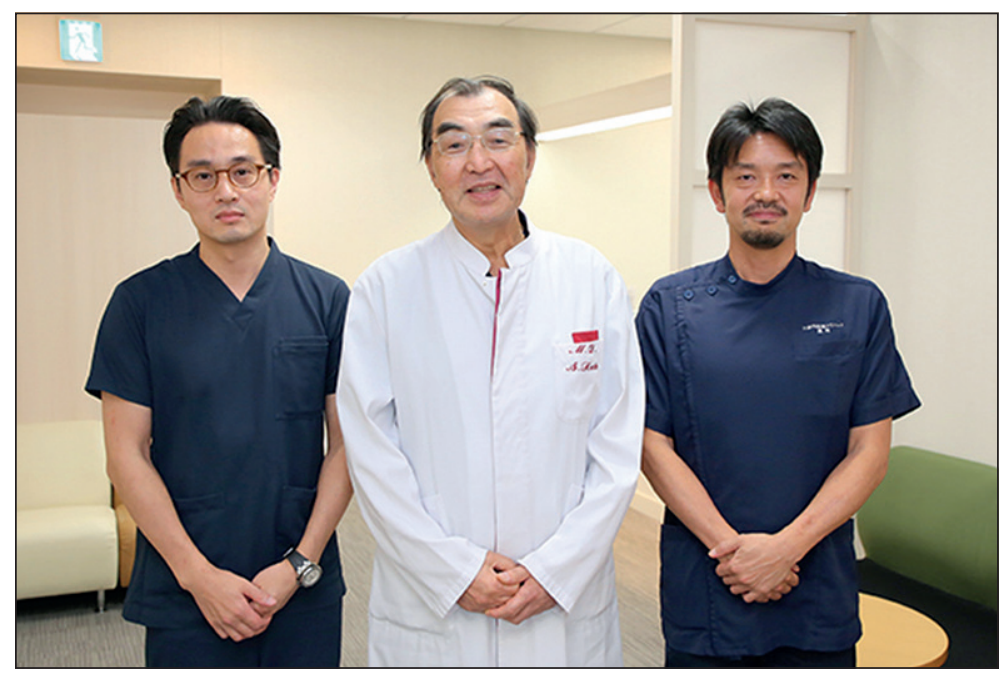

Figura 2. Su tarea de maestro, mentor y formador de generaciones de endoscopistas en Showa University- Yokohama- Japón.

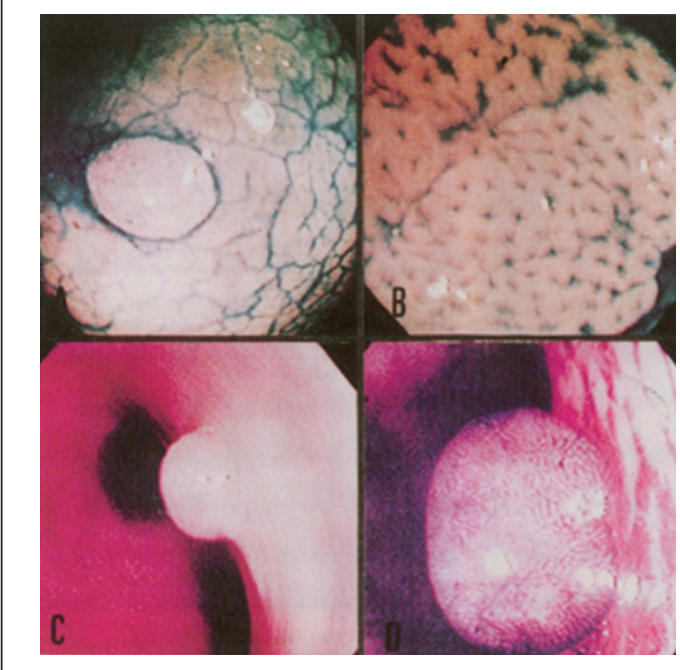

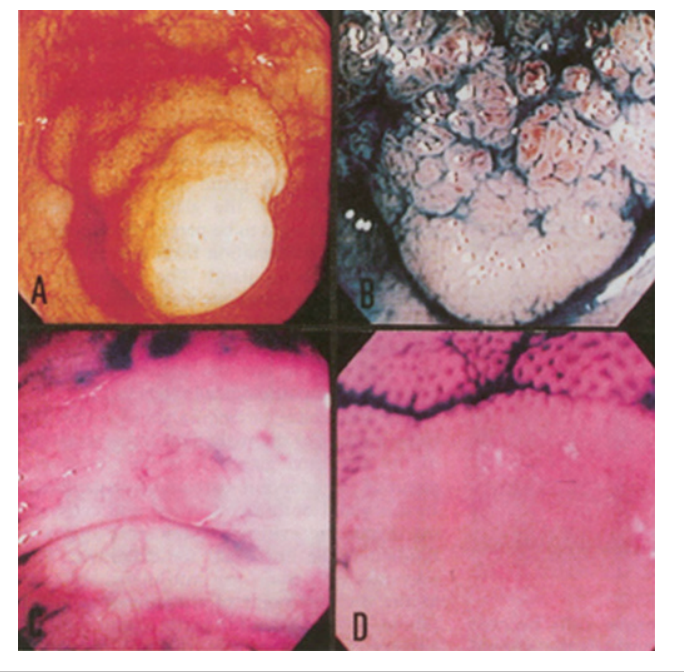

Figura 3. Imágenes "Pit Pattern" en neoplasia colorrectal visión con endoscopia magnificada. "Endoscopy 2001 


\section{Gastroenterología y algo más}

Figura 4. Profesor Shin-ei-Kudo en una demostración con casos en vivo en USA.

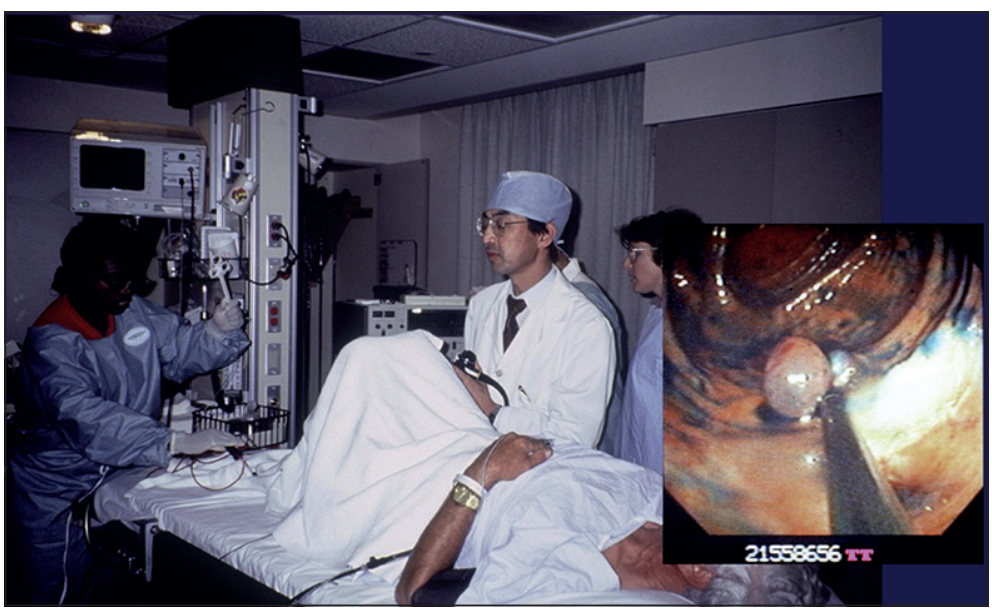

\section{Referencias}

1. Dr. Shin-ei Kudo. Video on line www. VideoGIE.org.

2. Mori Y, Kudo SE, Berzin TM, Misawa M, Takeda K. Computer-aided diagnosis for colonoscopy. Endoscopy. 2017;49(8):813-819.

3. Kudo S, Hirota S, Nakajima T, Hosobe S, Kusaka H, Kobayashi T, Himori M, Yagyuu A. Colorectal tumours and pit pattern. J Clin Pathol. 1994 Oct; 47(10):880-5.

4. Kudo S, Rubio CA, Teixeira CR, Kashida $\mathrm{H}$, Kogure E. Pit pattern in colorectal neoplasia: endoscopic magnifying view. Endoscopy. 2001;33 (4):367-73.

5. Vinsard DG, Misawa M, Kudo SE, Rastogi A, Baqci U, Rex DK, et al. Quality Assurance of computer-aided Detection and Diagnosis in Colonoscopy Gastrointest Endosc 2019 Mar.

6. Patel R, Scuba B, Soetikno R, Kaltenbach T. Colorectal polyp characterization: Is my computer better than me? Endosc Int Open. 2018;6(3):E279-80.

7. Sano Y, Tanaka S, Kudo SE, Saito S, Matsuda T, Wada Y, et al. Narrow-band imaging (NBI) magnifying endoscopic classification of colorectal tumors proposed by the Japan NBI Expert Team. Dig Endosc 2016;28(5):526-33.

8. Misawa M, Kudo SE, Mori Y, Cho T, Kataoka S, Yamauchi A, et al. Artificial Intelligence-Assisted Polyp Detection for Colonoscopy: Initial Experience. Gastroenterology. 2018;154 (8):2027-9.

9. Kudo SE, Mori Y, Misawa M, Takeda $\mathrm{K}$, Kudo T, Itoh $\mathrm{H}$, et al. Artificial intelligence and colonoscopy: Current status and future perspectives. .Dig Endosc. 2019;31(4):363-71.

10. Iwatate M, Sano Y, Tanaka S, Kudo SE, Saito S, Matsuda T, et al; Validation study for development of the Japan NBI Expert Team classification of colorectal lesions. Japan NBI Expert Team (JNET). Dig Endosc. 2018;30 (5):642-651.

11. Vinsard DG, Mori Y, Misawa M, Kudo SE, Rastogi A, Bagci U, et al. Quality assurance of computer-aided detection and diagnosis in colonoscopy. Gastrointest Endosc. 2019;90(1):55-63.
12. Kudo SE, Misawa M, Mori Y, Hotta K, Ohtsuka K, Ikematsu H, et al. Artificial Intelligence-assisted System Improves Endoscopic Identification of Colorectal Neoplasms .Clin Gastroenterol Hepatol. 2020;18(8):1874-1881.

13. Ichimasa K, Kudo SE, Mori Y, Misawa M, Matsudaira S, Kouyama Y, et al. Artificial intelligence may help in predicting the need for additional surgery after endoscopic resection of T1 colorectal cancer. Endoscopy. 2018;50(3):230-240.

14. Kobayashi Y, Kudo SE, Miyachi H, Hosoya T, Ikehara N, Ohtsuka K, et al. Clinical usefulness of pit patterns for detecting colonic lesions requiring surgical treatment. Int J Colorectal Dis. 2011;26(12):1531-40.

15. Wada Y, Kudo SE, Misawa M, Ikehara N, Hamatani S. Vascular pattern classification of colorectal lesions with narrow band imaging magnifying endoscopy. Dig Endosc. 2011;23 Suppl 1:106-11. 